\title{
五芳基取代环戊二烯衍生物的合成及其聚集诱导荧光性质研究
}

\author{
邱淑海 $a, b$ 许胜杰 $b$ 周国富 ${ }^{a}$ 水玲玲 $*, a$ 朱晓张 $*, b$ \\ ( ${ }^{a}$ 华南师范大学华 南先进光电子研究院彩色电子纸显示技术研究所 广州 510006) \\ $\left({ }^{b}\right.$ 中国科学院化学研究所有机固体院重点实验室 北京 100190)
}

\begin{abstract}
摘要 五芳基取代环戊二烯衍生物是一类具有聚集诱导荧光性质的化合物, 但相应的研究比较少. 为了研究不同推拉 电子能力的取代基以及取代位置对聚集诱导荧光性质的影响和拓展其在化学传感器、生物探针等方面的应用, 我们合 成了不同芳香取代基的五芳基取代环戊二烯及其衍生物，并对其进行了紫外吸收、苂光光谱、荧光量子产率、单晶分 析等方面的测试表征, 重点研究了其聚集诱导荧光性质.
\end{abstract}

关键词 五芳基取代环戊二烯; 聚集诱导荧光

\section{Synthesis of 1,2,3,4,5-Pentaarylcyclopenta-2,4-diene Derivatives and Their Aggregation-Induced Emission Properties}

\author{
Qiu, Shuhai $^{a, b} \quad \mathrm{Xu}$, Shengjie $^{b} \quad$ Zhou, Guofu $^{a} \quad$ Shui, Lingling ${ }^{*, a} \quad$ Zhu, Xiaozhang ${ }^{*, b}$ \\ ( ${ }^{a}$ Electronic Paper Display Institute, South China Academy of Advanced Optoelectronics, South China Normal University, \\ Guangzhou 510006) \\ $\left({ }^{b}\right.$ CAS Key Laboratory of Organic Solids, Institute of Chemistry, Chinese Academy of Sciences, Beijing 100190)
}

\begin{abstract}
Pentaarylated cyclopentcyclodienes have aggregation-induced emission (AIE) characteristics and can be easily derivatized, but are rarely studied. In order to examine the effect of the electronic properties and the position of the substituents on AIE properties, an expedient method for the synthesis of a series of pentaarylated cyclopentadiene derivatives was developed, and their UV-Vis, fluorescence spectra, fluorescence quantum yields as well as the XRD structures of single crystals were investigated. The AIE properties of pentaarylated cyclopentadiene derivatives were studied in detail.

Keywords pentaarylated cyclopentadienes; aggregation-induced emission
\end{abstract}

聚集诱导荧光，顾名思义，是由于分子聚集而产生 的荧光现象 ${ }^{[1 \sim 3]}$. 大多数发光分子在良性溶剂中可以发 出强烈的光, 但是在不良溶剂或固态时分子会形成聚集

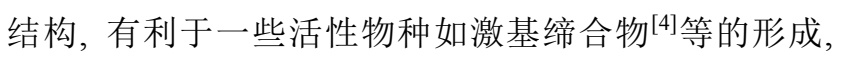
从而导致荧光猝灭 ${ }^{[5]}$. 但是, 具有聚集诱导荧光现象的 分子却恰好与此相反, 当这些分子溶解在良性溶剂时, 几乎看不到苂光; 而在不良溶剂或者固态时却由于聚集 而产生很强的苂光. 这种聚集诱导荧光现象可以解释为 由于分子内取代基运动受到限制而引起的结果 ${ }^{[6]}$.

2001 年, 唐本忠课题组 ${ }^{[1]}$ 发现分子聚集可以将 1-甲 基-1,2,3,4,5-五苯基硅咯从一个弱的发光分子转变为一
种很强的发光物质, 这种结果与聚集猝灭发光的常规现 象正好相反. 在随后的十多年里, 聚集诱导荧光材料引 起了人们的广泛关注与研究, 在有机发光二极管、生物 传感及成像、苂光染料、化学传感、生物探针等领 域 ${ }^{[711]}$ 表现出很好的应用前景. 在具有聚集诱导荧光性 质的分子中, 硅咯在有机发光二极管 ${ }^{[12]}$ 和传感 ${ }^{[13]}$ 领域 表现出优异的发光性能, 然而硅咯类化合物合成相对复 杂, 合成成本较高 ${ }^{[14]}$, 这在一定程度上影响了其应用. 五芳基取代环戍二烯与硅咯类化合物有着相似的分子 结构, 根据 $\mathrm{HF} / 6-31 \mathrm{G}^{*}$ 的计算结果, 五芳基取代环戊二 烯的最高占据分子轨道(HOMO)比硅咯高 $0.4 \mathrm{eV}$, 最低

\footnotetext{
*E-mail: shuill@scnu.edu.cn; xzzhu@iccas.ac.cn

Received February 26, 2015; revised March 30, 2015; published online April 3, 2015.

Project supported by the National Natural Science Foundation of China (Nos. 91333113, 21303060), the National Basic Research Program of China (973 Program, No. 2014CB643502), the Strategic Priority Research Program of the Chinese Academy of Sciences (No. XDB12010200), and the Guangdong Natural Science Foundation (No. S2013010014418).

国家自然科学基金(Nos. 91333113, 21303060)、国家重点基础研究发展计划(973 计划, No. 2014CB643502)、中国科学院先导 B 专项(No. XDB12010200)、广东省自然科学基金(No. S2013010014418)资助项目.
} 
空分子轨道(LUMO)高 $1.3 \mathrm{eV}^{[15]}$. 基于环戊二烯结构的

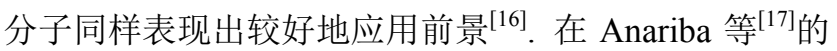
研究工作中, 五苯基取代环戊二烯化合物在甲苯中具有 高达 0.69 的荧光量子产率, 在水溶液中荧光量子产率也 达到了 0.14. 由于大多数聚集诱导苂光分子都具有疏水 芳香环, 表现出较差的水溶性, 五苯基取代环戊二烯衍 生物在水相中表现出较好的聚集荧光现象, 进一步拓展 了聚集诱导荧光材料的应用范围. 在我们的工作中, 我 们发展了简单的合成方法合成了一系列五芳基取代环 戊二烯及其衍生物, 进一步研究了不同推拉电子能力及 不同取代位置的取代基对聚集诱导荧光性质的影响.

\section{1 结果与讨论}

\section{1 五芳基取代环戊二烯的合成}

五芳基取代环戊二烯的合成可以通过钯催化环戊 二烯的芳基化反应实现. 化合物 $3 \mathbf{a} \sim \mathbf{3 d}$ 根据之前报道 的文献合成 ${ }^{[18]}$. 化合物 $3 \mathrm{e} \sim 3 \mathrm{~h}$ 的合成采用类似的钯催 化环戊二烯的方法, 用三叔丁基膦作为配体, 碳酸铯作 为碱, $N, N^{\prime}$-二甲基甲酰胺(DMF)作为溶剂, 在氮气保护 下 $130{ }^{\circ} \mathrm{C}$ 反应 1 6 h, 即可分离得到产品, 如 Eq. 1 所 示. 实验结果显示, 当芳基上含有较强给电子能力的取 代基时, 反应有较好的产率; 虽然对氟溴苯具有中等产 率 $(60 \%)$, 但是对于大多数拉电子基团而言, 产率非常 低, 甚至不反应. 化合物 $\mathbf{3 e}$ 和 $\mathbf{3 g}$ 的产率仅为 $25 \%$, 对于 拉电子能力比较强的三氟甲基溴苯反应时, 只能在环戊 二烯三个碳上实现取代; 对于甲酸甲酯基、氰基、硝基 等, 基本不反应. 取代基的位阻也会对反应有较大的影 响, 当使用甲苯基取代环戊二烯时, 虽然间、对位有较 好的产率, 但邻位却有很多副产物, 产率很低. 当使用 2,6-二甲基溴苯反应时, 只得到三取代产物; 当芳香基 为 3,5-二叔丁基苯基时, 只得到了 1,2,3,4-位取代的四取 代化合物. 另外, 该反应对杂环芳香基也有一定的选择 性, 当使用 3-溴噻吩反应时, 可以获得较好的产率 $(3 \mathbf{h}$, $56 \%$ ), 但是使用 2-溴噻吩、2-溴吡啶时, 反应却无产物 生成.

\section{2 五芳基取代环戊二烯醇的合成}

目前合成五芳基取代环戊二烯醇最常见的方法如 Eq. 2 所示 ${ }^{[19]}$, 这种方法对反应底物的要求较高. 如 Eq. 3 所示, 我们发展了新的简便合成方法. 五芳基取代环 戊二烯的羟基化在碱性条件下与空气接触室温即可反 应, 只是反应速率比较低. 将五芳基取代环戊二烯溶于 $\mathrm{DMF}$, 加入碳酸铯并加热到 $80{ }^{\circ} \mathrm{C}$, 在 $1 \sim 2 \mathrm{~h}$ 内就有 90\%左右的环戊二烯发生了 1-位羟基化. 进一步简化实 验操作, 我们在环戊二烯的芳基取代反应结束后, 直接 将温度降至 $80{ }^{\circ} \mathrm{C}$, 并将反应体系与空气接触 $1 \sim 2 \mathrm{~h}$,
便可实现产物的羟基化. 羟基化产物的反应结果跟环戊 二烯芳基化的结果相似, 对于给电子能力比较强的基 团, 反应有比较好的产率, 如 $\mathbf{4 a} \sim \mathbf{4 c} 、 \mathbf{4 f}$ 所示. 另外, 对 氟苯基及 3-噻吩基也有较好的产率.

\section{3 五芳基取代环戊二烯的紫外吸收、荧光性质}

为了研究不同取代基对聚集荧光性质的影响, 我们 测量了五芳基取代环戊二烯在二氯甲烷及粉末状态时 的紫外吸收和荧光谱图. 如图 1a 所示, 化合物 $\mathbf{3 a} \sim \mathbf{3 e}$ 在粉末状态时, 随着取代基供电子能力的增强发生红 移, 其最大紫外吸收波长依次为 344 (3d), 352 (3e), 352 (3a), 354 (3b), $358(\mathbf{3 c}) \mathrm{nm}$, 除 3e 外, 其最大荧光发射波 长也随取代基供电子能力的增强发生红移, 如图 $1 \mathrm{~b}$ 所 示. 3e 在薄膜状态时的最大苂光波长位置为 $455 \mathrm{~nm}$, 在 粉末状态时为 $475 \mathrm{~nm}$, 谱图明显变宽, 表明在粉末状态 时可能形成了激基缔合物. 另外，通过各化合物的紫外 吸收谱图的比较(如辅助材料中图 S3、S4 所示), 可以发 现取代基的位置对其紫外吸收也有一定影响.
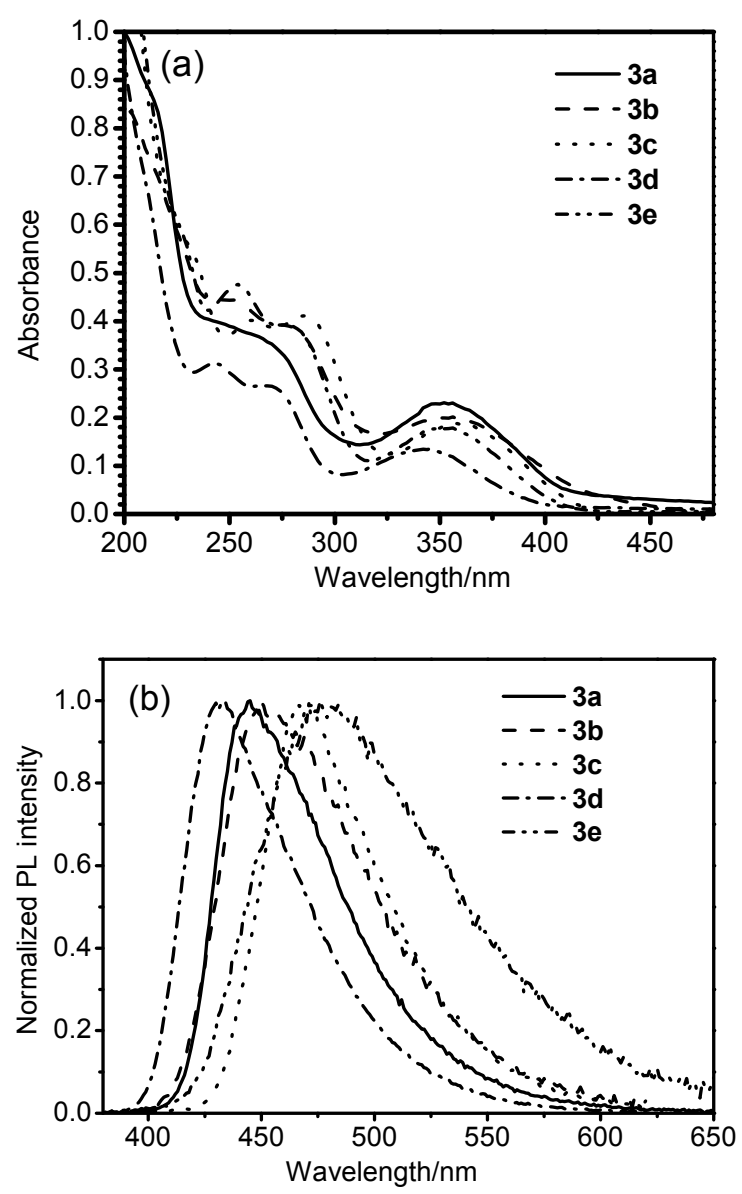

图 1 (a)不同芳基取代环戊二烯化合物 3a 3e 在粉末状态时 的紫外吸收谱图; (b)粉末状态时的苂光光谱

Figure 1 (a) UV-Vis absorption of compounds $3 \mathbf{a} \sim \mathbf{3 e}$ in the powders; (b) Fluorescence spectra compounds $\mathbf{3 a} \sim \mathbf{3 e}$ in the powders 


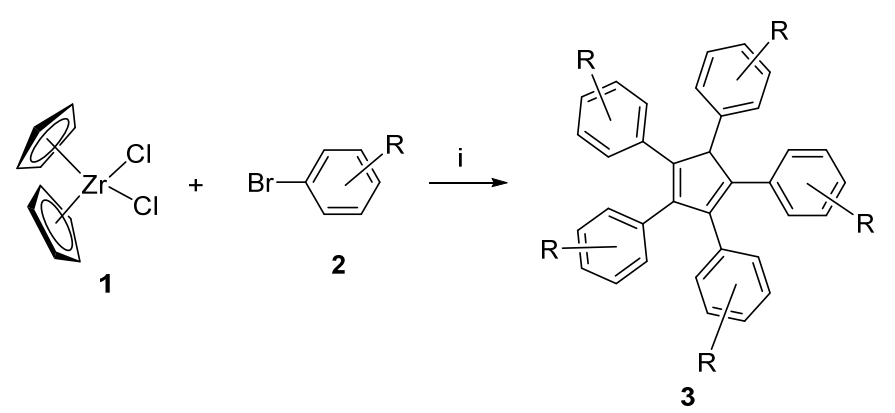

(1)

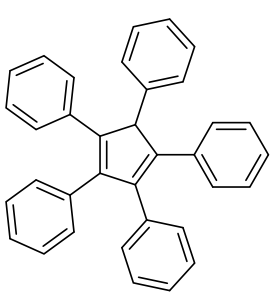

3a: $77 \%$

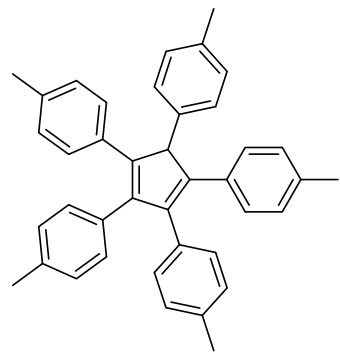

3b: $78 \%$<smiles>COc1ccc(C2=C(c3ccc(OC)cc3)C(c3ccc(OC)cc3)C(c3ccc(OC)cc3)=C2c2ccc(OC)cc2)cc1</smiles>

3c: $45 \%$<smiles>Fc1ccc(C2=C(c3ccc(F)cc3)C(c3ccc(F)cc3)C(c3ccc(F)cc3)=C2c2ccc(F)cc2)cc1</smiles>

3d: $60 \%$<smiles>Clc1ccc(C2=C(c3ccc(Cl)cc3)C(c3ccc(Cl)cc3)C(c3ccc(Cl)cc3)=C2c2ccc(Cl)cc2)cc1</smiles>

3e: $25 \%$<smiles>COc1cccc(C2=C(c3cccc(OC)c3)C(c3cccc(OC)c3)C(c3cccc(OC)c3)=C2c2cccc(OC)c2)c1</smiles>

3f: $64 \%$<smiles>Fc1cccc(C2=C(c3cccc(F)c3)C(c3cccc(F)c3)C(c3cccc(F)c3)=C2c2cccc(F)c2)c1</smiles>

3g: $25 \%$<smiles>c1cc(C2=C(c3ccsc3)C(c3ccsc3)C(c3ccsc3)=C2c2ccsc2)cs1</smiles>

3h: $56 \%$

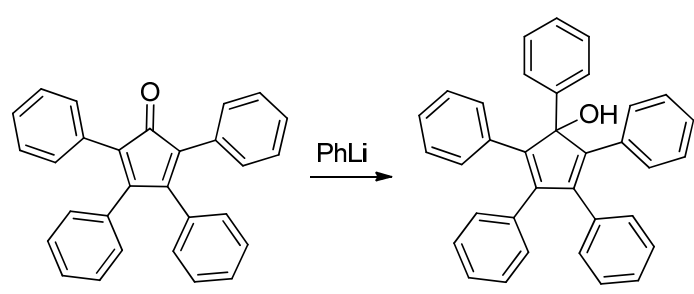

五芳基取代环伐二烯化合物 3a $~ 3 \mathrm{~g}$ 均表现出典型 的聚集诱导荧光现象, 以 $3 \mathrm{a}$ 为例, 在不同混合比例的四 氢呋喃一水溶液中, 呈现出不同的荧光现象, 如图 2 所 示. 当水含量在 $60 \%$ 以下时, 几乎看不到苂光, 但是当 水含量达到 $70 \%$ 时, 苂光强度急剧升高, 当水含量增加 到 $80 \%$ 、 $90 \%$ 时，我们发现溶液的荧光逐渐减弱. 通过 粒径仪测得的数据(如辅助材料中图 S6 所示), 我们发现 随着水含量的变化, 化合物 3a 聚集尺寸也在发生变化. 当水含量在 $60 \%$ 以上时, 聚集结构尺寸迅速增加, 但随 着水含量进一步提高, 聚集尺寸逐渐减小, 说明混合溶 液中聚集荧光受到颗粒尺寸大小的影响 ${ }^{[20]}$. 除了苂光 强度的变化, 随着水含量的增加, 相应的荧光发射位置
也发生一定变化. 在水含量为 $70 \% 、 80 \%$ 和 $90 \%$ 时，苂 光发射位置分别为 $437 、 440$ 和 $451 \mathrm{~nm}$, 表明随着水含 量的增加，其相应的苂光会发生红移.

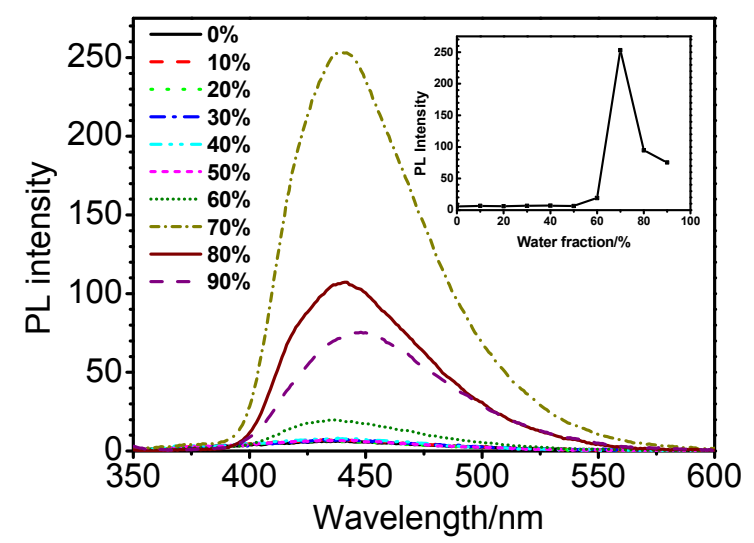

图 2 化合物 3a 在不同比例四氢呋喃-水混合溶液中的苂光光 谱图

Figure 2 PL spectra of compound 3a in THF-water mixtures Concentration of 3a: $10 \mu \mathrm{mol} \cdot \mathrm{L}^{-1}$; Excitation wavelength: $352 \mathrm{~nm}$ 

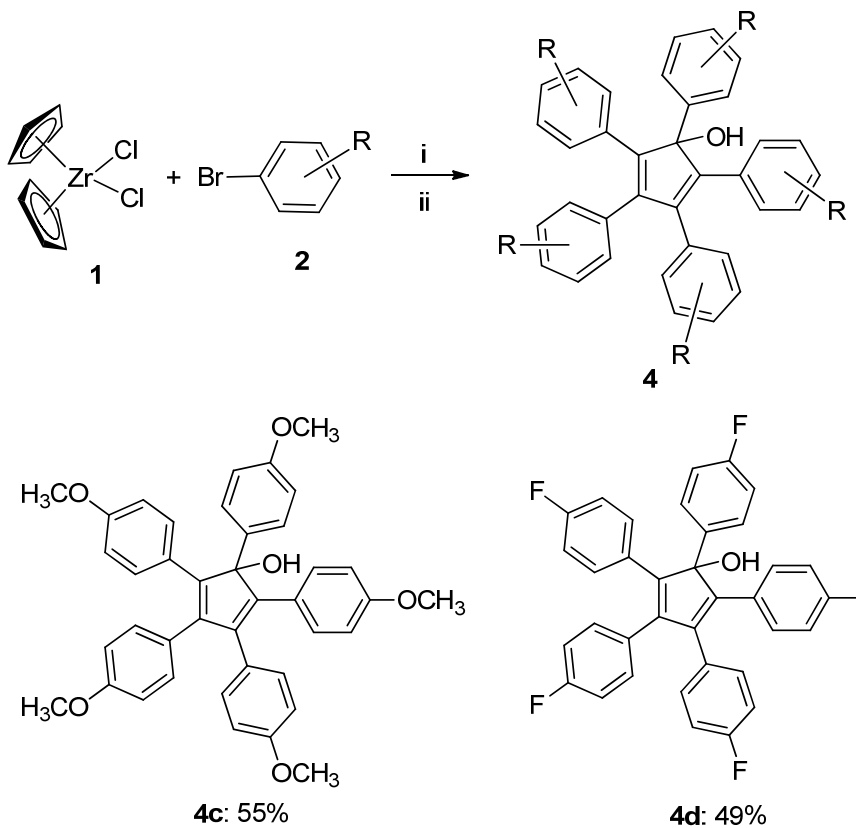

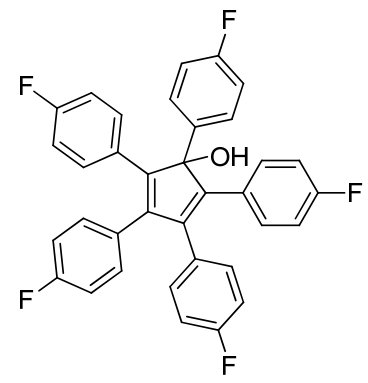

4d: $49 \%$

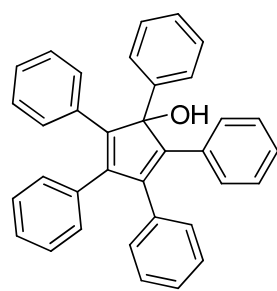

4a: $66 \%$

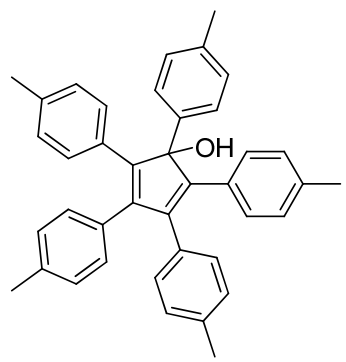

4b: $72 \%$<smiles>COc1cccc(C2=C(c3cccc(OC)c3)C(O)(c3cccc(OC)c3)C(c3cccc(OC)c3)=C2c2cccc(OC)c2)c1</smiles>

4f: $67 \%$

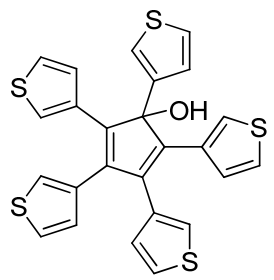

4h: $69 \%$

Reagents and conditions: (i) $\mathrm{Pd}(\mathrm{OAc})_{2}, \mathrm{P}(t-\mathrm{Bu})_{3}, \mathrm{Cs}_{2} \mathrm{CO}_{3}$, DMF, $130{ }^{\circ} \mathrm{C}$; (ii) Air, $80{ }^{\circ} \mathrm{C}$

\section{4 固体荧光量子产率}

为了研究不同芳香取代基及五元环结构对五芳基 取代环戊二烯类化合物光学性质的影响, 我们进一步合 成了一系列化合物 $\mathbf{5} \sim \mathbf{8}$, 并测量了各化合物苂光量子 产率数据, 结果如表 1 所示. 表中所有的化合物溶解在 二氯甲烷中时表现出非常弱的苂光, 但是在粉末状态时 大多数化合物都发射出较强的荧光. 为了研究不同五元 环骨架对聚集诱导荧光性质的影响, 我们首先对硅咯、 环戊二烯、吡咯相应化合物的固态荧光量子产率做了比 较. 1-甲基-五苯基硅咯 ${ }^{[15]}$ 在薄膜状态的苂光量子产率为 $85 \%$ ，1-甲基-五苯基环戊二烯(6)的苂光量子产率为 $31 \%$. 两类化合物具有相似的紫外吸收、苂光发射特点， 但是环戊二烯类的化合物的荧光量子产率要相对低一 些，除了与分子轨道能级有关 ${ }^{[16]}$ 外，可能还与不同的原 子半径有关. 通过 3a 与五苯基吡咯 (8)比较, 我们发现 芳基取代环戊二烯类化合物要比吡咯类的化合物有更 好的聚集诱导荧光效果, 这与吡咯化合物的 LUMO 能 级的交错分布有关 ${ }^{[20(a)]}$. 通过更换芳基上的取代基, 发 现对于大多数取代基而言, 不管是吸电子的还是给电子 的, 除含氟取代基外, 对苂光量子产率都有着负面的影 响, 这在一定程度上说明取代基对聚集荧光的影响主要 是空间效应. 产物 3c、3f、4c、4 f 的苂光量子产率相对 于 $3 \mathbf{a} 、 4 \mathbf{a}$ 有较大程度的下降, 这可能是由于甲氧基具有 一定柔性, 导致分子内运动比较容易, 荧光相对较弱; 而产物 3d、4d 表现出一定程度上的荧光量子产率的提 高, 这可能是由于吸电子的氟基与相邻分子芳基上的氢 形成了 $\mathrm{F}-\mathrm{H}$ 键, 从而使分子排列更加紧密, 分子内的
运动更加困难 ${ }^{[18]}$. 因此在不同的取代基中，含氟芳基取 代的环戊二烯化合物具有最好的聚集荧光效果. 另外, 通过对 5-(4-氯丁氧基)-1,2,3,4,5-五苯基环戊二烯(5)、5甲基-1,2,3,4,5-五苯基环戊二烯(6)、5-甲氧基-1,2,3,4,5五苯基环伐二烯(7)比较发现，1-位基团的取代对聚集荧 光效果也有一定影响. 化合物 $\mathbf{5}$ 与 $\mathbf{4 a}$ 相比, 并没有荧 光量子产率的损失, 这一定程度上证明了 4 系列化合物 在聚集荧光应用方面的潜力.

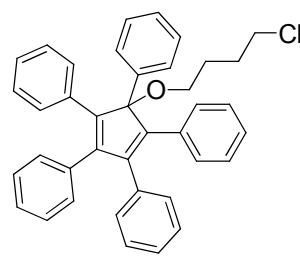

5

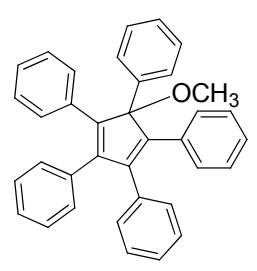

7

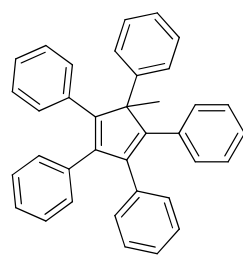

6

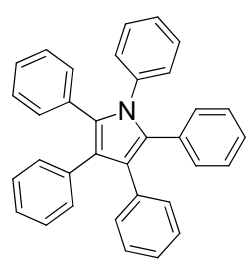

8

\section{5 单晶结构解析}

通过二氯甲烷/正己烷的混合溶液缓慢挥发的方式 生长化合物 $\mathbf{4 a}$ 的单晶, 有趣的是, 我们得到了两种不同 聚集结构的单晶, 如图 3 所示. 两种单晶呈现出不同的 颜色, 一种为黄色透明晶体, 在 $365 \mathrm{~nm}$ 激发时呈现出淡 黄色荧光，分子结构如图 3a 所示; 另一种为无色透 
表 1 五芳基取代环戊二烯衍生物的光物理性质

Table 1 Optical properties of 1,2,3,4,5-Pentaarylcyclopentadiene Derivatives

\begin{tabular}{|c|c|c|c|c|}
\hline \multirow{2}{*}{ 化合物 } & \multicolumn{2}{|c|}{$\lambda_{\mathrm{abs}}^{\max }$} & \multirow{2}{*}{$\begin{array}{c}\lambda_{\mathrm{em}}^{\max } \\
\text { Solid/nm }\end{array}$} & \multirow{2}{*}{$\begin{array}{l}\text { 苂光量子 } \\
\text { 产率 } b^{\circ} \%\end{array}$} \\
\hline & Soln ${ }^{a} / \mathrm{nm}$ & Solid $^{b} / \mathrm{nm}$ & & \\
\hline $3 \mathbf{a}$ & 340 & 352 & 445 & 38.0 \\
\hline $3 b$ & 346 & 354 & 450 & 21.1 \\
\hline $3 c$ & 356 & 358 & 468 & 21.4 \\
\hline 3d & 338 & 344 & 434 & 46.4 \\
\hline $3 e$ & 346 & 352 & 476 & 16.2 \\
\hline $3 f$ & 348 & 350 & 465 & 16.1 \\
\hline $3 g$ & 340 & 346 & 438 & 32.1 \\
\hline $3 \mathrm{~h}$ & 352 & 362 & 444 & 1.4 \\
\hline $4 a$ & 356 & 374 & 460 & 16.0 \\
\hline $4 b$ & 358 & 370 & 476 & 29.3 \\
\hline $4 c$ & 370 & 376 & 482 & 3.7 \\
\hline $4 d$ & 352 & 358 & 443 & 33.3 \\
\hline $4 f$ & 358 & 370 & 485 & 8.1 \\
\hline $4 h$ & 358 & 376 & - & 0.3 \\
\hline 5 & 354 & 370 & 457 & 19.0 \\
\hline 6 & - & 335 & 426 & 31.1 \\
\hline 7 & 350 & 370 & 457 & 12.5 \\
\hline 8 & - & 302 & 379 & 14.7 \\
\hline
\end{tabular}

${ }^{a} \operatorname{soln}$ 代表化合物在二氯甲烷溶液中的测量结果, 浓度为 $1 \times 10^{-5} \mathrm{~mol} \cdot \mathrm{L}^{-1}$; ${ }^{b}$ solid 代表为无定形粉末测量得到的数据结果.

明晶体, 在 $365 \mathrm{~nm}$ 激发时呈现出蓝色苂光, 分子结构如 图 3b 所示. 化合物的固态堆积方式对其荧光性质具有

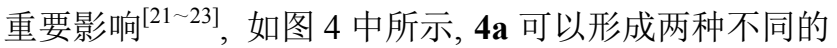
晶体堆积结构, 黄色晶体(对应于图 3a) 为单斜晶体, 相 邻分子间存在多重苯环 $\pi-\pi$ 相互作用, 间距为 $3.8 \AA$ (如 图 4 中虚线框所示); 无色晶体(对应于图 3b)由于 $\mathbf{4 a}$ 与 水分子的氢键作用, 形成三斜晶体, 分子间不存在明显 的 $\pi-\pi$ 作用. 此外黄色晶体中, $\mathbf{4 a}$ 呈现相对平面的分子构 象，更有利于 $\pi$ 电子离域 $[2,5$ 位取代苯基与环戊二烯间 二面角为： $24.87^{\circ}$ 和 $30.33^{\circ}$ (黄色晶体), $43.12^{\circ}$ 和 $51.11^{\circ}$ (无色晶体)], 综上, 我们推测两种晶体的颜色和 苂光差异可能是由于不同的分子排列方式和构象所引 起的. 通过比较两种晶体的分子堆积结构, 我们发现晶 体的堆积方式对荧光颜色的调控有着十分重要的影响. a

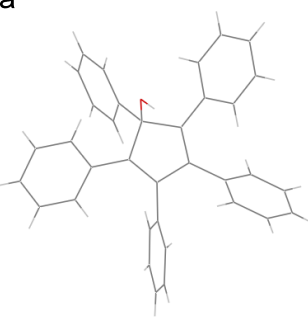

b

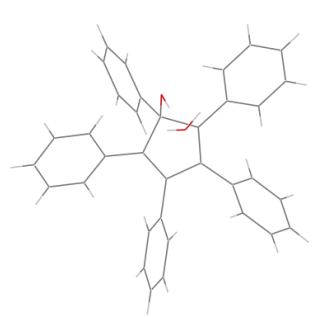

图 3 产物 $4 \mathbf{a}$ 的两种晶体分子结构

Figure 3 Two different molecule structures of compound $\mathbf{4 a}$
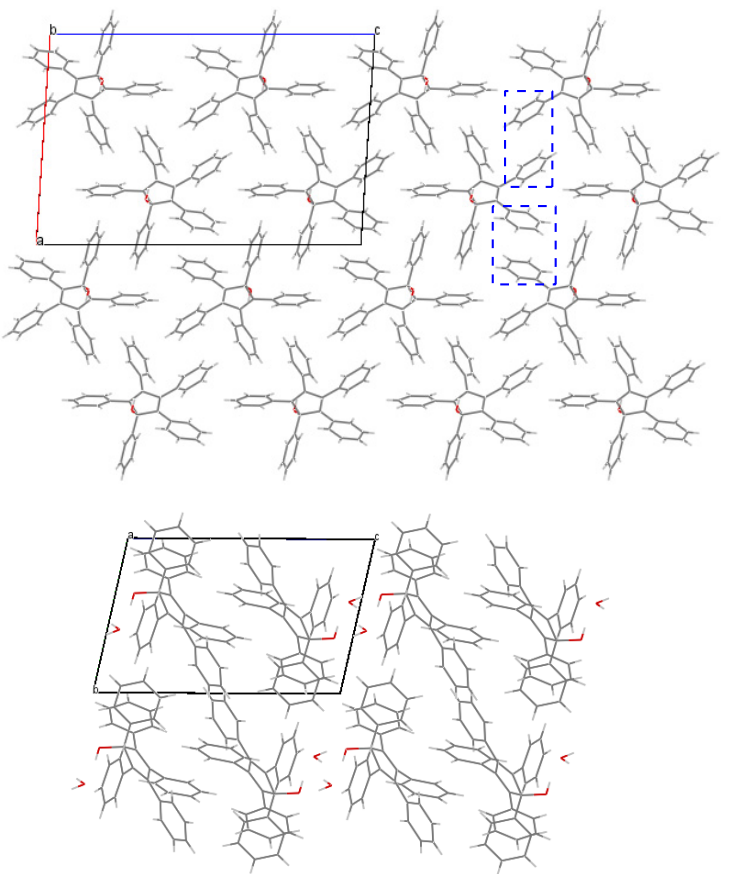

图 4 化合物 $4 \mathrm{a}$ 的两种不同堆积结构

Figure 4 Two different stacking images of compound 4a

\section{2 结论}

我们发展了钯催化合成五芳基取代环戊二烯醇化 合物的简便合成方法，合成了一系列五芳基取代环戊二 烯衍生物。通过紫外吸收、荧光光谱、苂光量子产率等 测试发现，随着芳基上取代基团供电子能力增强，紫外 吸收及苂光发生相应地红移; 大多数取代基对聚集荧光 有着负面的影响，但含氟芳基可以产生最好的聚集诱导 荧光效果. 通过单晶解析发现, 五苯基取代环戊二烯醇 可以通过与水分子共结晶形成不同于本体的晶体结构 和聚集诱导苂光现象.

\section{3 实验部分}

\section{1 仪器与试剂}

对空气或水分敏感的反应均在氮气或氩气保护下 在干燥反应管中进行，对空气或水分敏感的配体均使用 用注射器移取. 薄层层析用覆盖有 $0.25 \mathrm{~mm}$ 厚, 200 300 目硅胶的玻璃板，由烟台德信生物科技有限公司生 产，通过紫外灯进行分析. 有机溶剂通过旋转蒸发仪减 压除去. 双环戊二烯二氯化锆购自百灵威公司，三叔丁 基膦(10\%正已烷溶液)购自北京伊诺凯科技有限公司， 4 -氯溴苯从北京国药试剂购买, 醋酸钯、碳酸铯、对溴 甲苯、4-甲氧基溴苯、间溴甲苯、间甲氧基澳苯、间氟 溴苯均购自北京偶和科技公司, 对氟溴苯购自东京化成 公司. 其余均为市售分析纯试剂. 干燥 $N, N$-二甲基甲酰 
胺通过氢化钻减压蒸馏获得. ${ }^{1} \mathrm{H}$ NMR 和 ${ }^{13} \mathrm{C}$ NMR 用 Bruker Ultrashield Plus 型 $300 \mathrm{MHz}$ 或 $400 \mathrm{MHz}$ 核磁共振 仪测得, 以 $\mathrm{CDCl}_{3}$ 为溶剂, TMS 作内标. 基质辅助激光 解析电离飞行时(MALDI-TOF-MS)测试在 Bruker 质谱 仪上进行. 紫外-可见吸收光谱在 Shimadzu UV-3600 型紫外-可见分光光度计上测得, 荧光发射光谱在 Shimadzu RF-5301PC 苂光光谱仪上测得, 绝对苂光量 子产率在带有 F-3018 积分球的 NANO-FL3-1066C 苂光 光谱仪上测得. 混合溶液中的聚集粒径数据是由仪器 NanoPlus Zeta/nano Particle Analyzer 测得.

\section{2 实验方法}

\subsection{1 五芳基取代环戊二烯化合物的合成}

化合物 3a 3i 按照文献[18]报道方法合成, 具体实 验操作如下所示: 氮气保护下, 向 $15 \mathrm{~mL}$ 的耐压管内的 干燥 $N^{\prime}, N$-二甲基甲酰胺中依次加入醋酸钯 $(14 \mathrm{mg}$, $0.0625 \mathrm{mmol}) 、$ 三叔丁基膦 $(10 \%$ 正己烷溶液, $0.6 \mathrm{~mL}$, $0.25 \mathrm{mmol}) 、$ 碳酸铯 $(0.98 \mathrm{~g}, 3.0 \mathrm{mmol})$ 、双环戊二烯二氯 化锆 $(74 \mathrm{mg}, 0.25 \mathrm{mmol}$ )和溴化芳烃 $(3 \mathrm{mmol})$, 然后密封 置于 $130{ }^{\circ} \mathrm{C}$ 的油浴锅内, 反应 $1 \sim 6 \mathrm{~h}$. 冷却到室温后过 滤, 用二氯甲烷洗涤沉淀. 滤液用隔膜泉在 $65{ }^{\circ} \mathrm{C}$ 减压 除去, 然后用二氯甲烷溶解并加入适量硅胶, 再次减压 除去溶剂，用硅胶快速层析柱分离得到产品.

1,2,3,4,5-五对氯苯基环戊二烯 (3e): 淡黄色固体, 产率为 $25 \% .{ }^{1} \mathrm{H} \mathrm{NMR}\left(300 \mathrm{MHz}, \mathrm{CDCl}_{3}\right) \delta: 4.93(\mathrm{~s}, 1 \mathrm{H})$, $6.82(\mathrm{~d}, J=8.6 \mathrm{~Hz}, 4 \mathrm{H}), 6.89(\mathrm{~d}, J=8.5 \mathrm{~Hz}, 4 \mathrm{H}), 7.01 \sim$ $7.10(\mathrm{~m}, 6 \mathrm{H}), 7.14 \sim 7.18(\mathrm{~m}, 6 \mathrm{H}) ;{ }^{13} \mathrm{C}$ NMR $(100 \mathrm{MHz}$, $\left.\mathrm{CDCl}_{3}\right) \delta: 62.2,128.5,128.7,129.3,129.7,130.2,131.4$, 133.1, 133.5, 133.6, 135.6, 143.2, 146.1; MS $m / z$ (\%): 618 $\left(\mathrm{M}^{+}, 100\right), 620$ (64), 616 (63). Anal. calcd for $\mathrm{C}_{35} \mathrm{H}_{21} \mathrm{Cl}_{5}: \mathrm{C}$ 67.93, H 3.42; found C 67.59, H 3.50.

1,2,3,4,5-五间甲氧基环戊二烯(3f): 白色固体, 产 率为 $74 \% .{ }^{1} \mathrm{H}$ NMR $\left(400 \mathrm{MHz}, \mathrm{CDCl}_{3}\right) \delta: 3.46(\mathrm{~s}, 6 \mathrm{H})$, $3.55(\mathrm{~s}, 6 \mathrm{H}), 3.71(\mathrm{~s}, 3 \mathrm{H}), 5.02(\mathrm{~s}, 1 \mathrm{H}), 6.50 \sim 6.61(\mathrm{~m}$, $6 \mathrm{H}), 6.61 \sim 6.74(\mathrm{~m}, 7 \mathrm{H}), 6.78(\mathrm{~s}, 1 \mathrm{H}), 6.87(\mathrm{~d}, J=7.5 \mathrm{~Hz}$, $1 \mathrm{H}), 6.96(\mathrm{t}, J=7.9 \mathrm{~Hz}, 2 \mathrm{H}), 7.01 \sim 7.15(\mathrm{~m}, 3 \mathrm{H}) ;{ }^{13} \mathrm{C}$ NMR (75 MHz, $\left.\mathrm{CDCl}_{3}\right) \delta: 54.9,55.2,111.6,113.1,113.2$, $113.8,114.5,115.1,121.4,121.7,122.7,128.7,129.1$, 129.6, 137.0, 137.6, 139.8, 144.1, 146.2; MS $m / z$ (\%): 596 $\left(\mathrm{M}^{+}, 100\right), 597$ (44), 598 (10). Anal. calcd for $\mathrm{C}_{40} \mathrm{H}_{36} \mathrm{O}_{5}: \mathrm{C}$ 80.51, H 6.08; found C 80.37, H 6.10 .

1,2,3,4,5-五间氟苯基环伐二烯(3g): 白色固体, 产 率为 $25 \% .{ }^{1} \mathrm{H}$ NMR $\left(400 \mathrm{MHz}, \mathrm{CDCl}_{3}\right) \delta: 5.02(\mathrm{~s}, 1 \mathrm{H})$, $6.63(\mathrm{~d}, J=10.5 \mathrm{~Hz}, 2 \mathrm{H}), 6.67 \sim 6.94(\mathrm{~m}, 12 \mathrm{H}), 7.02$ (dt, $J=17.2,7.8 \mathrm{~Hz}, 3 \mathrm{H}), 7.17(\mathrm{td}, J=14.3,8.1 \mathrm{~Hz}, 3 \mathrm{H}) ;{ }^{13} \mathrm{C}$ NMR $\left(75 \mathrm{MHz}, \mathrm{CDCl}_{3}\right) \delta: 62.3(\mathrm{~d}, J=6.1 \mathrm{~Hz}), 114.2(\mathrm{~d}$,
$J=84.2 \mathrm{~Hz}), 114.4(\mathrm{~d}, J=83.8 \mathrm{~Hz}), 114.7(\mathrm{~d}, J=83.7 \mathrm{~Hz})$, $114.8(\mathrm{~d}, J=86.1 \mathrm{~Hz}), 115.7$ (d, $J=88.4 \mathrm{~Hz}), 116.8$ (d, $J=$ $86.5 \mathrm{~Hz}), 124.4(\mathrm{~d}, J=11.0 \mathrm{~Hz}), 124.8(\mathrm{~d}, J=11.1 \mathrm{~Hz})$, $125.8(\mathrm{~d}, J=11.3 \mathrm{~Hz}), 129.6$ (d, $J=33.8 \mathrm{~Hz}), 130.0(\mathrm{~d}, J=$ $33.5 \mathrm{~Hz}), 130.5$ (d, $J=33.3 \mathrm{~Hz}), 137.0(\mathrm{~d}, J=32.0 \mathrm{~Hz})$, $137.3(\mathrm{~d}, J=31.7 \mathrm{~Hz}), 139.4(\mathrm{~d}, J=17.7 \mathrm{~Hz}), 143.6(\mathrm{~d}$, $J=7.8 \mathrm{~Hz}), 146.1(\mathrm{~d}, J=9.4 \mathrm{~Hz}), 160.8,161.1,161.5$, 164.1, 164.3, 164.8; MS m/z (\%): $536\left(\mathrm{M}^{+}, 100\right), 537$ (38), 538 (7). Anal. calcd for $\mathrm{C}_{35} \mathrm{H}_{21} \mathrm{~F}_{5}$ : C 78.35, H 3.95; found C 77.98, H 3.97.

1,2,3,4,5-五(3-噻吩)环戊二烯(3h): 黄色固体, 产率 为 $56 \% .{ }^{1} \mathrm{H}$ NMR $\left(400 \mathrm{MHz}, \mathrm{CDCl}_{3}\right) \delta: 5.07(\mathrm{~s}, 1 \mathrm{H}), 6.66$ (dd, $J=5.0,1.0 \mathrm{~Hz}, 2 \mathrm{H}), 6.77 \sim 6.78(\mathrm{~m}, 2 \mathrm{H}), 6.87$ (td, $J=$ 9.3, 5.2, $3.7 \mathrm{~Hz}, 5 \mathrm{H}), 7.03(\mathrm{dd}, J=5.0,3.0 \mathrm{~Hz}, 2 \mathrm{H}), 7.17$ (dd, $J=4.8,3.0 \mathrm{~Hz}, 1 \mathrm{H}), 7.17$ (dd, $J=4.8,3.0 \mathrm{~Hz}, 1 \mathrm{H}$ ); ${ }^{13} \mathrm{C}$ NMR $\left(75 \mathrm{MHz}, \mathrm{CDCl}_{3}\right) \delta: 57.7,122.3,122.8,124.1$, $124.4,125.0,126.1,126.7,127.8,129.0,136.4,136.6$, 137.8, 139.0, 140.1; MS m/z (\%): $476\left(\mathrm{M}^{+}, 100\right), 477$ (27), 478 (23). Anal. calcd for $\mathrm{C}_{25} \mathrm{H}_{16} \mathrm{~S}_{5}: \mathrm{C} 62.99$, H 3.38; found C 62.75, H 3.30 .

\subsection{2 五芳基取代环戊二烯醇的合成}

氮气保护下, 向 $15 \mathrm{~mL}$ 耐压管内的干燥 $N^{\prime}, N$-二甲 基甲酰胺中依次加入醋酸钯 $(14 \mathrm{mg}, 0.0625 \mathrm{mmol})$ 、三叔 丁基膦 $(10 \%$ 正己烷溶液, $0.6 \mathrm{~mL}, 0.25 \mathrm{mmol}) 、$ 碳酸铯 $(0.98 \mathrm{~g}, 3.0 \mathrm{mmol})$ 、双环戊二烯二氯化锆 $(74 \mathrm{mg}, 0.25$ $\mathrm{mmol}$ )和溴化芳烃 $(3 \mathrm{mmol})$, 然后密封置于 $130{ }^{\circ} \mathrm{C}$ 的油 浴锅内, 反应 1 6 h. 体系温度降至 $80{ }^{\circ} \mathrm{C}$ 时, 打开瓶 盖, 继续摚拌 1 2 h. 反应体系完全冷却后过滤, 并用 二氯甲烷清洗沉淀. 滤液用隔膜百在 $65{ }^{\circ} \mathrm{C}$ 减压除去, 然后再用二氯甲烷溶解, 加入适量硅胶, 再次减压除去 溶剂, 然后用硅胶快速层析柱分离得到产品.

1,2,3,4,5-五对氟苯基环戊二烯醇(4d): 白色固体, 产率为 $49 \% .{ }^{1} \mathrm{H}$ NMR $\left(400 \mathrm{MHz}, \mathrm{CDCl}_{3}\right) \delta: 2.36(\mathrm{~s}, 1 \mathrm{H})$, $6.76(\mathrm{t}, J=8.7 \mathrm{~Hz}, 4 \mathrm{H}), 6.80 \sim 7.00(\mathrm{~m}, 14 \mathrm{H}), 7.45(\mathrm{dd}$, $J=8.7,5.4 \mathrm{~Hz}, 2 \mathrm{H}) ;{ }^{13} \mathrm{C}$ NMR $\left(75 \mathrm{MHz}, \mathrm{CDCl}_{3}\right) \delta: 89.8$, 115.1, 115.4, 115.6, 115.9, 126.7 (d, $J=31.8 \mathrm{~Hz}), 129.5$ (d, $J=13.71 \mathrm{~Hz}), 130.5$ (d, $J=14.04 \mathrm{~Hz}), 131.3$ (d, $J=31.5$ Hz), 131.7 (d, $J=31.8 \mathrm{~Hz}), 135.4$ (d, $J=13.2 \mathrm{~Hz}), 147.1$, 160.5 (d, $J=40.5 \mathrm{~Hz}), 163.8$ (d, $J=39.6 \mathrm{~Hz}) ; \mathrm{MS} m / z(\%)$ : $552\left(\mathrm{M}^{+}, 100\right), 553$ (38), 554 (7). Anal. calcd for $\mathrm{C}_{35} \mathrm{H}_{21} \mathrm{~F}_{5} \mathrm{O}$ : C 76.08, H 3.83; found C 75.73, H 3.82.

1,2,3,4,5-五间甲氧苯基环戊二烯醇 (4f): 淡黄色固 体, 产率为 $67 \% .{ }^{1} \mathrm{H}$ NMR $\left(300 \mathrm{MHz}, \mathrm{CDCl}_{3}\right) \delta: 2.53$ (s, $1 \mathrm{H}), 3.46(\mathrm{~s}, 6 \mathrm{H}), 3.52(\mathrm{~s}, 6 \mathrm{H}), 3.74(\mathrm{~s}, 3 \mathrm{H}), 6.52 \sim 6.53$ (m, $2 \mathrm{H}), 6.58 \sim 6.75(\mathrm{~m}, 11 \mathrm{H}), 6.96(\mathrm{td}, J=7.7,1.3 \mathrm{~Hz}$, 
2H), $7.06(\mathrm{t}, J=7.9 \mathrm{~Hz}, 2 \mathrm{H}), 7.19 \sim 7.20(\mathrm{~m}, 3 \mathrm{H}) ;{ }^{13} \mathrm{C}$ NMR (75 MHz, $\left.\mathrm{CDCl}_{3}\right) \delta: 54.9,55.2,55.3,90.0,111.3$, $112.5,113.7,113.9,114.2,114.9,117.6,122.2,122.5$, 128.9 , 129.2, 129.6, 135.0, 136.6, 142.2, 142.6, 147.7, 158.9, 159.3, 160.0; MS m/z (\%): $612\left(\mathrm{M}^{+}, 100\right), 613$ (43), 614 (11). Anal. calcd for $\mathrm{C}_{40} \mathrm{H}_{36} \mathrm{O}$ : C 78.41, H 5.92; found C 78.32, H 5.89.

1,2,3,4,5-五(3-噻吩)基环戊二烯醇(4h): 橘黄色固 体, 产率为 $69 \% .{ }^{1} \mathrm{H} \mathrm{NMR}\left(300 \mathrm{MHz}, \mathrm{CDCl}_{3}\right) \delta: 2.53(\mathrm{~s}$, $1 \mathrm{H}), 6.69$ (dd, $J=5.1,1.2 \mathrm{~Hz}, 2 \mathrm{H}), 6.78$ (dd, $J=5.0,1.2$ $\mathrm{Hz}, 2 \mathrm{H}), 6.87 \sim 6.95(\mathrm{~m}, 3 \mathrm{H}), 7.04(\mathrm{dd}, J=5.1,3.0 \mathrm{~Hz}$, $2 \mathrm{H}), 7.15 \sim 7.24(\mathrm{~m}, 5 \mathrm{H}), 7.55(\mathrm{dd}, J=3.0,1.2 \mathrm{~Hz}, 1 \mathrm{H})$; ${ }^{13} \mathrm{C}$ NMR $\left(75 \mathrm{MHz}, \mathrm{CDCl}_{3}\right) \delta: 88.3,123.8,124.3,124.7$, $125.3,125.4,126.4,128.0,128.9,134.1,135.6,136.0$, 142.1, 142.8; MS m/z (\%): $492\left(\mathrm{M}^{+}, 100\right), 493$ (31), 494 (24). Anal. calcd for $\mathrm{C}_{25} \mathrm{H}_{16} \mathrm{~S}_{5}$ : C 60.94, H 3.27; found $\mathrm{C}$ 60.76, H 3.32 .

\section{2 .3 化合物 5 8 的合成}

化合物 $7^{[24]}$ 和 $8^{[25]}$ 按照文献所用方法合成.

5-(4-氯丁氧基)-1,2,3,4,5-五苯基环戊二烯(5): 氮气 保护下, 用干燥的四氢呋喃溶解化合物 $4 \mathbf{a}(0.187 \mathrm{~g}, 0.40$ $\mathrm{mmol})$, 然后加入氢化钠 $(60 \%$ 矿物油中, $0.80 \mathrm{mmol})$, 室 温反应 $0.5 \mathrm{~h}$. 然后加入 1,4 -二氯丁烷(108 mg, 0.48 $\mathrm{mmol}$ ), 反应 $1 \mathrm{~h}$ 后, 加入四丁基碘化铵(18 mg, 0.048 $\mathrm{mmol}$ ), 室温摚拌 $2 \mathrm{~h}$. 加入适量饱和食盐水, 用二氯甲 烷萃取 3 次, 用无水硫酸镁干燥, 旋干溶剂, 过硅胶柱, 得到淡黄色固体，产率为 $32 \%$. ${ }^{1} \mathrm{H}$ NMR $(400 \mathrm{MHz}$, $\left.\mathrm{CDCl}_{3}\right) \delta: 1.61 \sim 1.80(\mathrm{~m}, 4 \mathrm{H}), 3.27(\mathrm{t}, J=6.3 \mathrm{~Hz}, 2 \mathrm{H})$, 3.69 (t, $J=5.5 \mathrm{~Hz}, 2 \mathrm{H}), 7.00$ (d, $J=7.6 \mathrm{~Hz}, 13 \mathrm{H}), 7.17$ (t, $J=6.3 \mathrm{~Hz}, 7 \mathrm{H}), 7.23$ (d, $J=7.8 \mathrm{~Hz}, 2 \mathrm{H}), 7.62$ (d, $J=7.4$ $\mathrm{Hz}, 2 \mathrm{H}) ;{ }^{13} \mathrm{C} \mathrm{NMR}\left(75 \mathrm{MHz}, \mathrm{CDCl}_{3}\right) \delta: 27.1,29.5,44.9$, $62.1,94.7,125.5,126.9,126.9,127.3,127.7,128.2,128.4$, $129.2,130.0,134.1,135.7,139.9,144.3,145.4 ; \mathrm{MS} \mathrm{m} / z$ (\%): $552\left(\mathrm{M}^{+}, 100\right), 553$ (43), 554 (32). Anal. calcd for $\mathrm{C}_{39} \mathrm{H}_{33} \mathrm{ClO}$ : C 84.68, H 6.01; found C 84.59, H 5.99.

5-甲基-1,2,3,4,5-五苯基环戌二烯(6): 氮气保护下, 用干燥的四氢呋喃溶解化合物 3a (160 mg, $0.36 \mathrm{mmol})$, $0{ }^{\circ} \mathrm{C}$ 下缓慢滴加 $1.6 \mathrm{~mol} \cdot \mathrm{L}^{-1}$ 正丁基锂正己烷溶液 $(0.25$ $\mathrm{ml}, 0.40 \mathrm{mmol})$, 室温下搅拌下 1 h. $0{ }^{\circ} \mathrm{C}$ 下滴加碘甲烷 $(27 \mu \mathrm{L}, 0.43 \mathrm{mmol})$, 搅拌 $2 \mathrm{~h}$. 用饱和硫代硫酸钠淬灭反 应, 二氯甲烷萃取 3 次, 用无水硫酸镁干燥有机相, 旋 干溶剂, 过硅胶柱, 得到白色粉末固体, 产率为 $90 \% .{ }^{1} \mathrm{H}$ NMR (400 MHz, $\left.\mathrm{CDCl}_{3}\right) \delta: 1.68(\mathrm{~s}, 3 \mathrm{H}), 6.75(\mathrm{t}, 4 \mathrm{H})$, $6.76 \sim 7.03(\mathrm{~m}, 16 \mathrm{H}), 7.18 \sim 7.34(\mathrm{~m}, 5 \mathrm{H}) ;{ }^{13} \mathrm{C}$ NMR $(75$ $\left.\mathrm{MHz}, \mathrm{CDCl}_{3}\right) \delta: 18.9,63.3,126.5,126.6,126.7,127.6$,
$127.7,128.6,129.9,130.3,136.0,136.3,139.8,142.3,153$; MS m/z (\%): $460\left(\mathrm{M}^{+}, 100\right), 461$ (39), 462 (7). Anal. calcd for $\mathrm{C}_{36} \mathrm{H}_{28}$ : C 93.87, H 6.13; found C 93.59, H 6.12.

辅助材料(Supporting Information) 所有目标化合物 的紫外菼光谱图、 ${ }^{1} \mathrm{H} N M R 、{ }^{13} \mathrm{C} N \mathrm{NMR}$ 图谱及单晶解析 数据. 这些材料可以免费从本刊网站(http://sioc-journal. $\mathrm{cn} /)$ 上下载.

\section{References}

[1] Luo, J.-D.; Xie, Z.-L.; Lam, J. W. Y.; Cheng, L.; Chen, H.-Y.; Qiu, C.-F.; Kwok, H.-S.; Zhan, X.-W.; Liu, Y.-Q.; Zhu, D.-B.; Tang, B.-Z. Chem. Commun. 2001, 18, 1740.

[2] Mao, W.-G.; Chen, K.; Ouyang, M.; Sun, J.-W.; Zhou, Y.-B.; Wang, Y.-S.; Song, Q.-B.; Zhang, C. Chin. J. Org. Chem. 2014, 34, 161 (in Chinese).

(毛文纲，陈康，欧阳密，孙璟玮，周永兵，王永胜，宋庆宝，张 诚, 有机化学, 2014, 34, 161.)

[3] Guan, C.-F.; Qian, Y. Chin. J. Org. Chem. 2014, 34, 537 (in Chinese). (管成飞, 钱鹰, 有机化学, 2014, 34, 537.)

[4] Birks, J. B. Rep. Prog. Phys. 1975, 38, 903.

[5] Hong, Y.-N.; Lam, J. W. Y.; Tang, B.-Z. Chem. Soc. Rev. 2011, 40, 5361.

[6] Mei, J.; Hong, Y.-N.; Lam, J. W. Y.; Qin, A.-J.; Tang, Y.-H.; Tang, B.-Z. Adv. Mater. 2014, 26, 5429.

[7] (a) Zhao, Z.-J.; Chan, C. Y. K.; Chen, S.-M.; Deng, C.-M.; Lam, J. W. Y.; Jim, C. K. W.; Hong, Y.-N.; Lu, P.; Chang, Z.-F.; Chen, X.-P.; Lu, P.; Kwok, H.-S.; Qiu, H.-Y.; Tang, B.-Z. J. Mater. Chem. 2012, $22,4527$.

(b) Huang, J.; Yang, X.; Wang, J.-Y.; Zhong, C.; Wang, L.; Qin, J.-G.; Li, Z. J. Mater. Chem. 2012, 22, 2478.

[8] (a) Mei, J.; Tong, J.-Q.; Wang, J.; Qin, A.-J.; Sun, J.-Z.; Tang, B.-Z. J. Mater. Chem. 2012, 22, 17063

(b) Mei, J.; Wang, Y.-J.; Tong, J.-Q.; Wang, J.; Qin, A.-J.; Sun, J.-Z.; Tang, B.-Z. Chem. Eur. J. 2013, 19, 612.

(c) Anariba, F.; Chng, L. L.; Abdullah, N. S.; Tay, F. E. H. J. Mater. Chem. 2012, 22, 19303.

(d) Yu, Y.; Feng, C.; Hong, Y.-N.; Liu, J.-Z.; Chen, S.-J.; Ng, K. M.; Luo, K.-Q.; Tang, B.-Z. Adv. Mater. 2011, 23, 3298.

[9] Li, H.-Y.; Zhang, X.-Q.; Zhang, X.-Y.; Yang, B.; Wei, Y. Colloids Surf., B 2014, 121, 347.

[10] Chen, X.-J.; Shen, X.-Y.; Guan, E.-J.; Liu, Y.; Qin, A.-J.; Sun, J.-Z.; Tang, B.-Z. Chem. Commun. 2013, 49, 1503.

[11] (a) Franklin, A.; Chng, L.-L.; Nur, S. A.; Francis, T. J. Mater. Chem. 2012, 22, 19303.

(b) Tong, H.; Hong, Y.; Dong, Y.; Haeussler, M.; Li, Z.; Lam, J. W. Y.; Dong, Y.; Sung, H. H. Y.; Williams, I. D.; Tang, B.-Z. J. Phys. Chem. B. 2007, 111, 11817.

[12] Chen, L.; Jiang, Y.-B.; Nie, H.; Lu, P.; Sung, H.-H. Y.; Williams, I. D.; Kwok, H.-S.; Huang, F.; Qin, A.-J.; Zhao, Z.-J.; Tang, B.-Z. Adv. Funct. Mater. 2014, 24, 3621.

[13] (a) Wang, M.; Zhang, D.-Q.; Zhang, G.-X.; Zhu, D.-B. Chem. Commun. 2008, 37, 4469.

(b) Huang, J.; Wang, M.; Zhou, Y.-Y.; Weng, X.-C.; Shuai, L.; Zhou, X.; Zhang, D.-Q. Bioorg. Med. Chem. 2009, 17, 7743.

(c) Liu, Z.-T.; Xue, W.-X.; Cai, Z.-X.; Zhang, G.-X.; Zhang, D.-Q. J. Mater. Chem. 2011, 21, 14487. 
[14] Tang, B.-Z.; Zhan, X.-W.; Yu, G.; Lee, P. P. S.; Liu, Y.-Q.; Zhu, D.-B. J. Mater. Chem. 2001, 11, 2974.

[15] Yu, G.; Yin, S.-W.; Liu, Y.-Q.; Chen, J.-S.; Xu, X.-J.; Sun, X.-B.; Ma, D.-G.; Zhan, X.-W.; Peng, Q.; Shuai, Z.-G.; Tang, B.-Z.; Zhu, D.-B.; Fang, W.-H.; Luo, Y. J. Am. Chem. Soc. 2005, 127, 6335.

[16] (a) Zhang, X.-D.; Ye, J.-W.; Xu, L.-F.; Yang, L.-J.; Deng, D.; Ning, G.-L. J. Lumin. 2013, 139, 28.

(b) Gu, X.-G.; Yao, J.-J.; Zhang, G.-X.; Yan, Y.-L.; Zhang, C.; Peng, Q.; Liao, Q.; Wu, Y.-S.; Xu, Z.-Z.; Zhao, Y.-S.; Fu, H.-B.; Zhang, D.-Q. Adv. Funct. Mater. 2012, 22, 4862.

(c) Wu, Y.-T.; Kuo, M.-Y.; Chang, Y.-T.; Shin, C.-C.; Wu, T.-C.; Tai, C.-C.; Cheng, T.-H.; Liu, W.-S. Angew. Chem., Int. Ed. 2008, 47, 9891.

(d) Chen, Z.; Zhang, J.; Song, M.; Yin, J.; Yu, G.-A.; Liu, S.-H. Chem. Commun. 2015, 51, 326.

[17] Anariba, F.; Chng, L. L.; Abdullah, N. S.; Tay, F. E. H. J. Mater. Chem. 2012, 22, 19303.

[18] Dyker, G.; Heiermann, J.; Miura, M.; Inoh, J. I.; Pivsa-Art, S.; Satoh, T.; Nomura, M. Chem.-Eur J. 2000, 18, 3426.

[19] Kanthak, M.; Muth, E.; Dyker, G. Eur. J. Org. Chem. 2010, 31, 6084.

[20] (a) Feng, X.-A.; Tong, B.; Shen, J.-B.; Shi, J.-B.; Han, T.-Y.; Chen,
L.; Zhi, J.-G.; Lu, P.; Ma, Y.-G.; Dong, Y.-P. J. Phys. Chem. B 2010, $114,16731$.

(b) Ouyang, M.; Yu, C.-H.; Zhang, Y.-J.; Hu, B.; Lu, X.-J.; Sun, J.-W.; Zhang, C. Acta. Phys.-Chim. Sin. 2012, 28, 2944 (in Chinese).

(欧阳密, 俞春辉, 张玉建, 胡森, 吕晓静, 孙璟玮, 张诚, 物理 化学学报, 2012, 28, 2944.)

(c) Tang, H.-Y.; Zeng, Y.; Li, Y.-Y.; Chen, J.-P.; Li, Y. Acta Chim. Sinica 2011, 69, 2241 (in Chinese).

(唐海云, 曾毅, 李迎迎, 陈金平, 李嫕, 化学学报, 2011, 6 , 2241.)

[21] Yan, D.-P.; Yang, H.-J.; Meng, Q.-Y.; Lin, H.-Y.; Wei, M. $A d v$ Funct. Mater. 2014, 24, 587.

[22] Chi, Z.-G.; Zhang, X.-Q.; Xu, B.-J.; Zhou, X.; Ma, C. P.; Zhang, Y.; Liu, S.-W.; Xu, J.-R. Chem. Soc. Rev. 2012, 41, 3878.

[23] Dong, Y.-J.; Xu, B.; Zhang, J.-B.; Tan, X.; Wang, L.-J.; Chen, J.-L.; Lv, H.-G.; Wen, S.-P.; Li, B.; Ye, L.; Zou, B.; Tian, W.-J. Angew. Chem., Int. Ed. 2012, 51, 10782.

[24] Cai, R.-R.; Huang, M.-M.; Cui, X.-L.; Zhang, J.-Y.; Du, C.-X.; Wu, Y.-S.; Wu, Y. J. RSC Adv. 2013, 3, 13140.

[25] Chen, X.-P.; Li, X.-H.; Wang, N.-N.; Jin, J.-S.; Lu, P.; Wang, Y.-G. Eur. J. Org. Chem. 2012, 4380.

(Li, L.; Fan, Y.) 